\title{
Mathematics Learning Instrument: A Correlation and Means Difference Comparison with Federal Accountability Algebra Test Scores
}

\author{
Jeffrey C. Lear \\ Whitehall-Coplay School District, PA, United States
}

\begin{abstract}
Mathematics learning outcomes have specific cognitive development implications [2], [9]. Key contributions in 'structure sense' studies build upon a theoretical manuscript detailing a conceptualization of 'thinking mathematically' using symbols (i.e. 'symbol sense') [1], [6], [12], [13]. Likewise, from a theoretical basis, this manuscript reports and extends an initial conference presentation of the instrument: Algebra Concept Inventory to Measure Metric Sense (ACIMMS) [14]. This paper includes the complete instrument as well as developmental details, particularly; (a) a definition of 'metric sense' [9], (b) specific roles of mini-experts in Second Generation Instructional Design (ID2) [11], and, (c) creates a mathematics instrument using 'metric sense' to inventory five subject specific domain knowledge base items [5]. Selected applications of the instrument's constructs are illustrated in equations conventionally found in trigonometry and statistics courses. Upper classmen from a United States high school form a sample for a correlation and means difference study between federal accountability exam scores and ACIMMS algebra instrument data. Confirmed are findings from a bounded literature review [9], that concurrent use of all five metric sense conceptualizations are not extensively utilized by secondary students in manipulating mathematical expressions [6]. Despite time discrepancies of up to three years between results of ACIMMS instrument data and independent federal algebra accountability exam scores, a significant positive correlation is found. Correct answers on each instrument question, which forms two respective groups of students' independent algebra accountability exam scores, reveal a clear need for students' persistent understanding of all five metric sense

\section{Introduction}

This theoretical manuscript presents a definition of metric sense using a metric space [12]. Mathematics learning requires a continual examination of conceptual understanding while executing each step of procedural approaches to problem solving [2]. Recent research has examined the effect of using brackets in procedural processes to group structures according to an order of operations [6]. Other studies compare written forms of expressions to examine student comprehension of structure (i.e. structure sense) [1], [10].

In this paper it is contended that procedural processes are concurrent with structure comprehension in making sense of mathematics [10], [13]. Theoretically, the inherent mathematical structure of a metric space will define the algebraic structures mathematics students engage when using procedures to rearrange groupings of symbols to solve problems [12]. Five horizontal integrated algebra knowledge base elements are hypothesized as concurrently necessary to simplify expressions, solve equations, and build skills in mathematics learning [2], [5]. The distance function relating elements of a metric space (e.g. variable representations among symbols in algebra) is used in an analysis to justify each integrated algebra knowledge base element. Findings include an Algebra Concept Inventory to Measure Metric Sense and two examples which extend the research constructs to post algebra structures (see Tables 3, 4, 2, and Figures 1a, 1b respectively). The instrument is consistent with current lines of inquiry in mathematics learning and may compliment instrumentation developed in studies in Newtonian physics [3], [4].
\end{abstract} conceptualizations. Recommendations are made for teaching and further mathematics learning investigations.

\section{Metric Space and Metric Sense}

The most familiar metric space used to develop elementary algebraic skills is the Euclidean Space [8]. The Euclidean plane (i.e. Cartesian Plane RxR) is used as a representation for learning algebraic 
concepts where two real number lines intersect at an origin. The representation provides access to instruction on two variables $\mathrm{x}$ and $\mathrm{y}$. The following definition of a metric space theoretically supports the development of instrument design and element horizontal integration into a specific mathematics domain knowledge base [5], [12].

A metric space is a set $\mathbf{R}$ with a distance function (the metric d) that, for every two points $x, y$ in $\mathbf{R}$, gives the distance between them as a nonnegative real number $d(x, y)$. A metric space must also satisfy

1. $d(x, y)=0$ if and only if $x=y$

2. $d(x, y)=d(y, x)$ and

3. the triangle inequality $\mathrm{d}(\mathrm{x}, \mathrm{y})+\mathrm{d}(\mathrm{y}, \mathrm{z})>=$ $\mathrm{d}(\mathrm{x}, \mathrm{z})$.

"A Euclidian Space may be viewed as a vector space with the usual metric (distance) explored in secondary mathematics education. This focus on mathematical conceptual understanding, a refinement of structure sense within mathematical thinking, herein termed metric sense; is hypothesized to be instrumental in building empirical knowledge in" ... mathematics instruction ... "and the assessment of learning outcomes" [8], [9].

\section{Metric sense as horizontal integrated algebra knowledge base elements}

A purpose of this paper is to specify algebra knowledge base elements impacting future knowledge production in mathematics learning. Measuring metric sense cognitive skills is inherently measuring proficiency in identifying and correctly interpreting metric space elements [9], [12]. The following delineates five measurable constructs of metric sense conceptualization (MS1-MS5) throughout each step in an equation and its procedural solution (see Table 1).

1. MS1 - identify all the unary, binary, or other operations

2. MS2 - identify the main operation (MO) of an expression within an equation; utilizing all the expression, not missing anything

3. MS3 - correctly execute the inverse operation resulting in an equivalent equation

4. MS4 - identify all positive and negative symbols in an expression as either only part of a coefficient of a term, or concurrently as a coefficient symbol and a binary operation (i.e. sum and difference)

Table 1. Algebraic example identifying all metric sense elements in each solution step

\begin{tabular}{|c|c|c|}
\hline $\begin{array}{l}\quad \frac{+x^{2}-4}{+10}=+6 \\
\begin{array}{l}\text { MS3 \{apply inverse, } \\
\text { multiply\} }\end{array}\end{array}$ & $\begin{array}{l}\text { MS2 MO quotient } \\
\text { MS5 } \\
\text { Quotient of (difference and 10) } \\
\qquad \\
\text { (Square and 4) } \\
\downarrow \\
\mathrm{x}\end{array}$ & $\begin{array}{l}\text { MS1 - Two binary operations }(-, /) \\
\text { and one unary operation (square) } \\
\text { MS4 - Three positive symbols as } \\
\text { coefficients on terms; one negative } \\
\text { symbol as symbol on } 4 \text { having dual } \\
\text { purpose as binary operation } \\
\text { difference/subtraction (total } 4 \text { ) }\end{array}$ \\
\hline MS3 \{apply inverse add $\}$ & $\begin{array}{l}\text { MS2 MO difference } \\
\text { MS5 } \\
\text { Difference (Square and 4) } \\
\qquad \\
\\
\text { x }\end{array}$ & $\begin{array}{l}\text { MS1 - One binary operation (-) and } \\
\text { one unary operation (square) } \\
\text { MS4 - Two positive symbols as } \\
\text { coefficients on terms; one negative } \\
\text { symbol as symbol on } 4 \text { having dual } \\
\text { purpose as binary operation } \\
\text { difference/subtraction (total 3) }\end{array}$ \\
\hline $\begin{array}{r}\text { MS3 \{apply inverse } \sqrt{x^{2}}= \\
\sqrt{64}\}\end{array}$ & $\begin{array}{ccc}\text { MS2 } & \text { MO } & \text { square } \\
& & \\
\text { MS5 } & & \\
& & \text { (Square) } \\
& \downarrow \\
& & \mathrm{x}\end{array}$ & $\begin{array}{l}\text { MS1 - one unary operation } \\
\text { MS4 - Two positive symbols as } \\
\text { coefficients on terms; no positive or } \\
\text { negative symbols having dual } \\
\text { purpose as binary operations (total 2) }\end{array}$ \\
\hline$+x=+8^{*}$ & & $\begin{array}{l}\text { MS4 - Two positive symbols as } \\
\text { coefficients on terms; no positive or } \\
\text { negative symbols having dual } \\
\text { purpose as binary operations (total 2) }\end{array}$ \\
\hline
\end{tabular}

Note. $*$ Positive result for illustration 
5. MS5 - identify in writing, the hierarchy order of operations beginning with the main operation (MO), throughout the entire expression, detailing all operands of unary and binary operations.

\section{A relationship between metric sense knowledge elements and a mathematical metric space}

The hypothesis of this manuscript is: a student who has a high level of metric sense in the five horizontal integrated algebra knowledge base elements has a knowledge base necessary to simplify expressions, solve equations, and build knowledge in a metric space setting.

\section{Theoretical analysis of the hypothesis}

Represented here are theoretical justifications for the hypothesis [12].

MS1, MS4: A metric space requires, for every two points $\mathrm{x}, \mathrm{y}$ in $\mathbf{R}$, the triangle inequality $\mathrm{d}(\mathrm{x}, \mathrm{y})+$ $\mathrm{d}(\mathrm{y}, \mathrm{z})>=\mathrm{d}(\mathrm{x}, \mathrm{z})$ holds. Therefore, the summation, and its inverse binary operation, difference, must be identifiable as independent or concurrent uses of the + and - symbols respective of an element's distance from either side of zero on a real number line.
MS2, MS5: Novotná and Hoch [12, p. 95] find "An important feature of structure sense is the substitution principle, which states that if a variable or parameter is replaced by a compound term (product or sum), or if a compound term is replaced by a parameter, the structure remains the same." Therefore, a main operation (MO) of an expression within an equation, utilizing all the expression, not missing anything; incorporates possible compound terms where in a metric space, for every two points $\mathrm{x}$, $\mathrm{y}$ in $\mathbf{R}$, gives the distance between them, $\mathrm{d}(\mathrm{x}, \mathrm{y})$.

MS3: The relationship between an operation's identity element and inverse elements of a metric space are identifiable for every element in the continuous set $\mathbf{R}$ [8].

An extended application of the five measurable components of metric sense conceptualization, with the same theoretical justifications, can be applied to post algebra mathematics learning [12]. An application of conceptualizing metric sense in a trigonometric equation is provided (see Table 2). Although introduced in algebra, it cannot be over emphasized that continual conceptualization and persistent application of the five horizontal integrated algebra knowledge base elements is critical to mathematics learning throughout secondary and tertiary education.

Table 2. Trigonometric example identifying metric sense knowledge base elements

\begin{tabular}{|c|c|c|l|}
\hline $\begin{array}{l}\text { Normally: } \\
\sin (\pi-x)=-2\end{array}$ & MS2 MO sin function \{unary\} & $\begin{array}{l}\text { MS1 - One binary operation (-) and } \\
\text { one unary operation, sin ( ) } \\
\text { MS4 - Three positive/negative } \\
\text { symbols as coefficients on terms, } \\
\text { One negative symbol as a symbol on } \\
\text { x with dual purpose as a binary } \\
\text { operation difference (total 4) }\end{array}$ \\
\hline Difference of
\end{tabular}

Note. $\sin ^{-1}$ is the inverse function of the sine function; first solution step provided for illustration

An additional application of metric sense and structure sense is presented for student learning on the proportional minimum sample size $\mathrm{n}$ formula from a familiar margin of error (E) equation (see Figure 1a and $1 \mathrm{~b}$ ). The classroom presentation episode is a successful example of turning theory into practice.

\section{A mathematics instrument using 'metric sense' to inventory algebra subject specific domain knowledge base elements}

Expressions typical in linear and quadratic equations are analyzed in ten questions of an Algebra Concept Inventory to Measure Metric Sense (see Table 3 and 4). Level appropriate vocabulary is 
Table 3. Algebra Concept Inventory to Measure Metric Sense p. 1

Answer questions 1-5 using the equation below:

$$
\frac{+2(+x-4)}{+3}+5=0 \quad \text { Normally } \quad \frac{2(x-4)}{3}+5=0
$$

1. Which is the main operation of the expression on the left side of the equation; utilizing all of the left expression, not missing anything, considering the order of operations
A. + symbol before the $x$
B. $\div$ division bar
C. + symbol before the 5
D. - symbol before the 4

2. Which is correct about the expression on the left side of the equation
A. four positive symbols on terms; one negative symbol also as subtraction (total five)
B. one positive symbol on terms; one negative symbol also as subtraction; three positive symbols also as addition (total five) symbol also as subtraction; two positive symbols also as addition (total five)
C. two positive symbols on terms; one negative
D. three positive symbols on terms; one negative symbol also as subtraction; one positive symbol also as addition (total five)

3. Which is accurate about the expression on the left side of the equation, considering the order of operations

A.

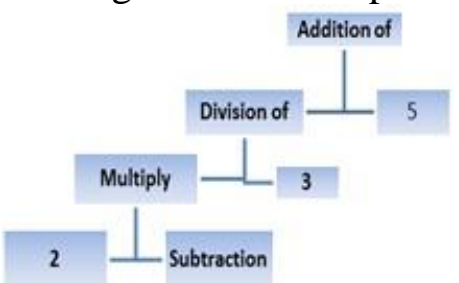

C.

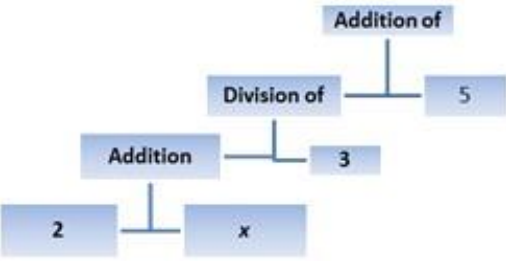

B.

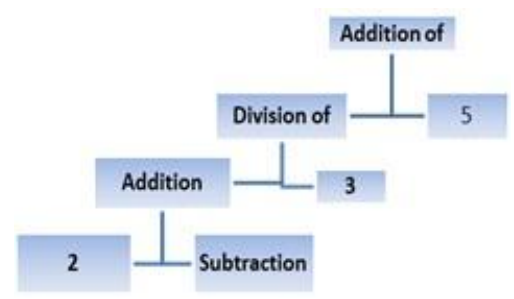

D

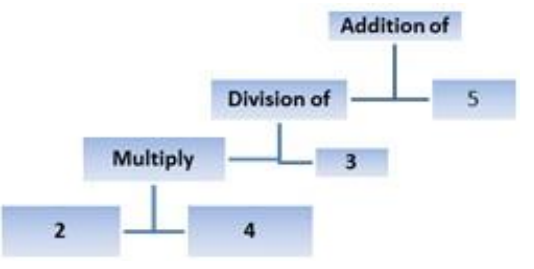

4. Which is accurate about the expression on the left side of the equation
A. It has one binary operation
B. It has four binary operations
C. It has five binary operations
D. It has no binary operations

5. What are the steps in solving the equation by reversing the main operation of the expression on the left side of the equation;
A. First subtract each side by 5 followed by multiplying by 3 as the next step
B. First subtract each side by 5 followed by adding by 4 as the next step
C. First subtract each side by 4 followed by multiplying by 3 as the next step
D. First add each side by 5 followed by dividing by 4 as the next step

Note. Coefficient symbols are shown and introductory vocabulary used for inventory instrument measurement.

Table 4. Algebra Concept Inventory to Measure Metric Sense p. 2 
Answer questions 6-10 using the equation below:

$$
-6+\sqrt{(-2)^{2}-4(+3)(-x)}=0 \quad \begin{aligned}
& \text { Normally } \\
& -6+\sqrt{(-2)^{2}-4(3)(-x)}=0
\end{aligned}
$$

6. Which is the main operation of the expression on the left side of the equation; utilizing all of the left expression, not missing anything, considering the order of operations
A. - symbol before the 4
B. + symbol after the 6
C. ${ }^{2}$ symbol (square)
D. $\sqrt{ }$ symbol (square root)

7. Which is correct about the expression on the left side of the equation
A. one negative symbol on terms; one positive symbol on terms; two positive symbols also as addition; two negative symbols also as subtraction (total six)
C. three negative symbols on terms; one positive symbol on terms; one positive symbol also as addition; one negative symbol also as subtraction (total six)
B. two negative symbols on terms; one positive symbol on terms; one positive symbol also as addition; two negative symbols also as subtraction (total six)
D. one negative symbol on terms; one positive symbol on terms; one positive symbol also as addition; three negative symbols also as subtraction (total six)

8. Which is accurate about the expression on the left side of the equation, considering the order of operations

A.

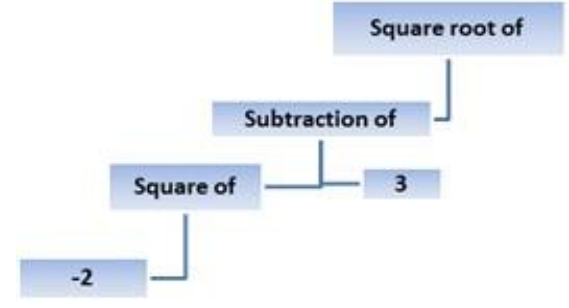

B.

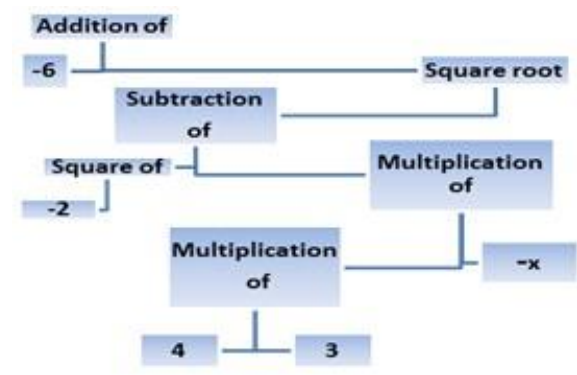

D.

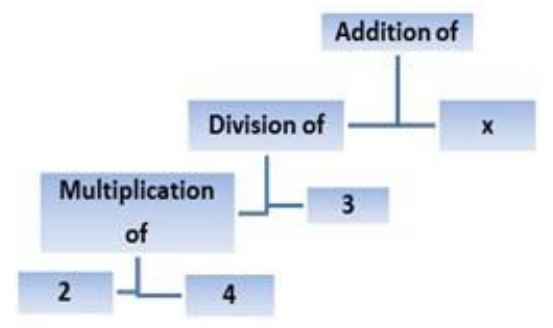

9. What is accurate about the expression on the left side of the equation
A. It has 6 binary and 1 unary operation
B. It has 4 binary and 1 unary operations
C. It has 1 binary and 4 unary operations
D. It has 4 binary and 2 unary operations

10. What are the steps in solving the equation by reversing the main operation of the expression on the left side of the equation;
A. First add each side by 6 followed by squaring each side as the next step
C. First add each side by -2 followed by squaring each side as the next step
B. First square each side followed by adding each side by 6
D. First square each side followed by adding each side by 2

Note. Coefficient symbols are shown and introductory vocabulary used for inventory instrument measurement. 

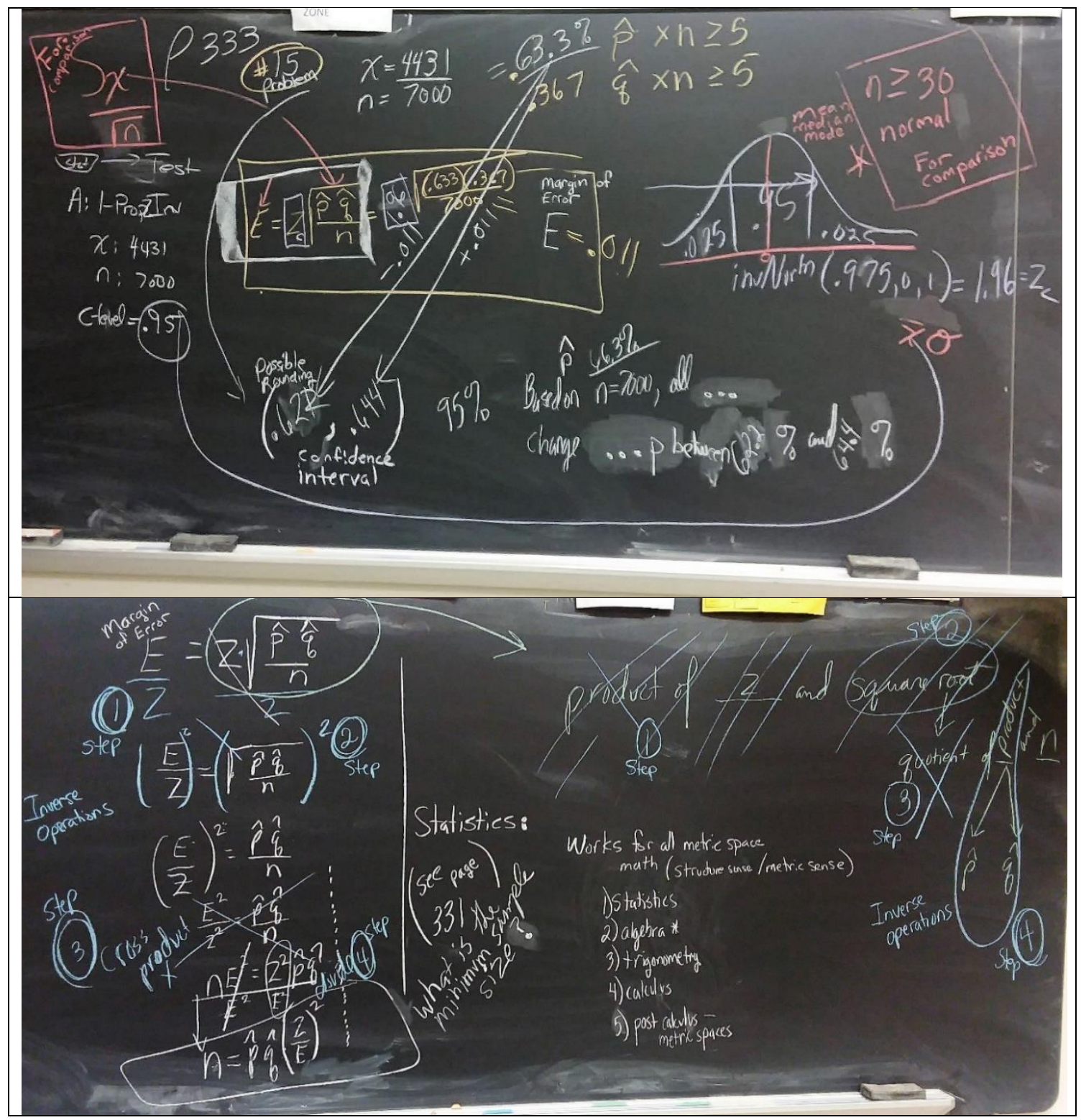

Figure 1a and $1 \mathrm{~b}$. Metric sense instruction as constructs on confidence intervals for proportions

integrated in the instrument which is conventionally familiar to beginning algebra students. Some latent coefficient symbols are present to accentuate structure sense knowledge base element identification while using the instrument (i.e. MS4, see Tables 1-4). It is recommended that instructors use the instrument customized and adapted to their individual needs. Instructors wishing to use the instrument to find a significance between data with other assessments in algebra may need to determine pre and post testing variables according to their particular school setting. Additional delineations may need to be specified when reporting on findings using the instrument.

\section{A sample for a correlation and means difference calculation}

Upper classmen from a United States high school provide sample data for a correlation and means difference study between their federal accountability algebra exam scores and the independent ACIMMS algebra instrument scores. Convenience sampling is used, as each student within the school has a school administrated federal accountability algebra exam score. Administrative permissions also warrant this sampling technique. 


\section{Methodology}

Approved by administration, students $(n=49)$ voluntarily participated in the instrument data collection, for credit, a few weeks before graduation as a normal mathematics activity included in their course work study. The majority of students received a federal accountability exam score up to three years prior, as a summative assessment at the conclusion of an algebra 1 course. The researcher determined that the time laps and participation in other mathematics courses allows for instrument measurement of retained algebraic metric sense understanding (i.e. use of the five algebra base elements MS1-MS5). Some vocabulary information was determined essential and was provided graphically as an additional page:

Unary Operations

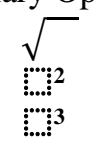

Binary Operations

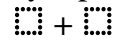

$$
\begin{aligned}
& \text { :...: } \\
& \text { :-...: } \\
& \ldots
\end{aligned}
$$

ACIMMS algebra instrument scores were recorded for each student, for each question. The instrument's range is $0-10$, one point for each of the 10 multiple choice question answered correctly. The State's federal accountability exam ranges for the following four categories, Below Basic, Basic, Proficient, and Advanced; are respectively, 1200-1438, 1439-1499, 1500-1545, 1546-1800. Each student's exam value was recorded. Student identification labels replaced any identifying information collected during the study, immediately after being paired. Paired data are plotted using a spreadsheet and a Pearson correlation coefficient critical value is used to determine correlation significance (see Figure 2). For each of the ten questions data of the ACIMMS instrument, based on correctness, two groups of accountability exam scores were created (see Table 5). Ten independent two samples mean t-test(s) assuming equal variances were calculated.

\section{Conclusions}

There are specific cognitive skills required in representing and explaining structure sense within the mathematical construct of a Euclidian Space [8], [9]. Students' skills can be quantified for instruction in manipulations of algebraic equations, and for formative or summative assessment. ACIMMS instrument scores and psychometrically valid federal accountability exam scores are significantly correlated, $r=.2418, p<.05$ (see Figure 2).

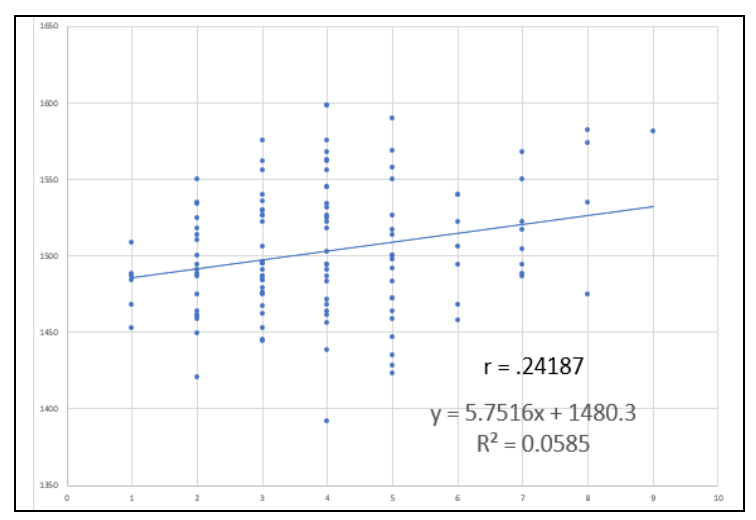

Figure 2. Paired data plot

Of the ten ACIMMS questions, only questions 4, 5, 9, and 10 had student responses greater than $50 \%$ correct (see Table 5). These responses are representative of MS1 and MS3 measurable constructs of metric sense conceptualization for linear and quadratic expressions. These are more identification tasked MS1 and procedural MS3 in students' interactions with expressions. The questions with student responses less than 50\% correct are representative of metric sense constructs MS2, MS4, and MS5. These require a more comprehensive understanding of entire expressions, including symbol location, orders of multiple operations, and symbol use comprehension (i.e. symbol sense and metric sense).

Table 5. Accountability exam scores

\begin{tabular}{lllll}
\hline & \multicolumn{3}{c}{ Correct } & \multicolumn{2}{c}{ Incorrect } \\
\cline { 2 - 5 } ACIMMS & $n$ & $M(S D)$ & $n$ & $M(S D)$ \\
\hline 1 & 8 & $1508.50(36.23)$ & 41 & $1520.02(43.32)$ \\
2 & 18 & $1524.11(46.06)$ & 31 & $1514.68(40.03)$ \\
3 & 12 & $1531.25(46.63)$ & 37 & $1513.89(40.32)$ \\
$4^{*}$ & 25 & $1530.04(46.59)$ & 24 & $1505.75(33.51)$ \\
5 & 36 & $1523.31(44.32)$ & 13 & $1503.85(32.64)$ \\
6 & 10 & $1512.80(37.37)$ & 39 & $1519.51(43.60)$ \\
7 & 21 & $1517.38(42.27)$ & 28 & $1518.71(42.77)$ \\
8 & 18 & $1512.22(38.69)$ & 31 & $1521.58(44.24)$ \\
9 & 28 & $1522.21(34.05)$ & 21 & $1512.71(51.37)$ \\
10 & 33 & $1517.27(40.67)$ & 16 & $1519.94(46.29)$ \\
\hline
\end{tabular}

Note. $*$ significance $t(1)=2.09, p<.05$.

Ten, two-samples mean t-test(s) assuming equal variances determined that for only half of the ACIMMS questions, the group of accountability exam scores corresponding to answering the question correctly, had a mean greater than the group mean representing the question incorrectly 
answered. It is notable that four of those correspond to linear expressions questions rather than quadratic expressions questions. The low correct to incorrect ratio for all questions responses may explain this accountability exam score result (see Table 5). It is also notable that the question with the highest correct : incorrect ratio is question $5,36: 13$. This is representative of students arriving at a procedural first step response MS3, but far from a success rate potential if they concurrently, persistently utilized all five instrument conceptualizations successfully.

ACIMMS questions 4, 5, and 9 are identified as corresponding to MS1 and MS3 measurable constructs of metric sense conceptualization for linear and quadratic expressions. Examination indicates that these are more identification tasked MS1 and procedural MS3 in students' interactions with expressions. For ACIMMS question number 4 (i.e. MS1 - identify all the unary, binary, or other operations), the mean for the group of accountability exam scores where the question was answered correctly $(M=1530.04, S D=46.59)$ reported significantly higher scores than the other group $(M$ $=1505.75, S D=33.51), t(1)=2.09, p<.05$. This significance is found, and may possibly be explained, where student focus is on how many binary $\left(+,-_{-}\right)$separations are between elements of binary operations, necessary in part, to begin with the first procedural step (i.e. question number 5) in manipulating a linear expression. The t-test results, like the direct ratio results, suggest that more comprehensive understanding and persistent use of symbol sense and metric sense is required when students interact with expressions.

\section{Implications for further research}

This manuscript provides the Algebra Concept Inventory to Measure Metric Sense (ACIMMS quantitative instrument) to measure mathematics conceptual understanding using five measurable constructs of a metric space. This inventory instrument was inspired by reviewing research manuscripts using the Force Concept Inventory (FCI) instrument [9]. The FCI test use in empirical studies has initiated substantial contributions to the line of inquiry in physics education [3], [4]. Instrumentation measuring the five constructs of metric sense conceptualization (MS1-MS5) has the potential to contribute similarly to the line of inquiry in mathematics education.

It is recommended that similar replication correlation studies verify the relationship between the variables of ACIMMS results and results commonly obtained through United States federal accountability exams. Replicating this study's two- samples independent means tests, under various initial field conditions, may reveal consistent conclusions that students emphasize procedures over metric sense comprehension when interacting with mathematical expressions. It is also recommended that mixed methods be implored along with the instrument where qualitative approaches can shed more light on students' use of ACIMMS metric sense constructs.

Included in mathematics learning mixed method studies, the instrument may provide evidence of relationships across data sources or research design approaches. It is also recommended the instrument be used with the FCI instrument to measure scientific reasoning ability; adding some clarity to unexplained negative correlation findings between specific physics learner sample subgroups [3].

Finally, it is recommended, and cannot be understated in the authors opinion, that instruction toward comprehension of MS1 through MS5 be concurrent and persistent in a comprehensive way until students can correctly generate tree illustrations as an expected outcome with extremely high success rates (see question numbers 3 and 8 and Figure $1 b$ ). It may be necessary to continue this practice throughout secondary and tertiary education for some mathematics students to routinely inspect expressions by thinking mathematically [13].

\section{References}

[1] Arcavi, A. (1994). Symbol sense: Informal sensemaking in formal mathematics. For the learning of Mathematics, 14(3), 24-35.

[2] Baumert, J., Kunter, M., Blum, W., Brunner, M., Voss, T., Jordan, A., Klosmann, U., Krauss, S., Neubrand, M., \& Tsai, Y. M. (2010). Teachers' mathematical knowledge, cognitive activation in the classroom, and student progress. American Educational Research Journal, 47(1), 133-180.

[3] Coletta, V. P., \& Phillips, J. A. (2005). Interpreting FCI scores: Normalized gain, preinstruction scores, and scientific reasoning ability. American Journal of Physics, 73(12), 1172-1182.

[4] Coletta, V. P., Phillips, J. A., \& Steinert, J. (2012). FCI normalized gain, scientific reasoning ability, thinking in physics, and gender effects. American Institute of Physics Conference Series, 1413, 23-26.

[5] Fischer, H. E., Klemm, K., Leutner, D., Sumfleth, E., Tiemann, R., \& Wirth, J. (2005). Framework for empirical research on science teaching and learning. Journal of Science Teacher Education, 16(4), 309-349. 
[6] Hoch, M., \& Dreyfus, T. (2004). Structure sense in high school algebra: The effect of brackets. In Proceedings of the 28th Conference of the International Group for the Psychology of Mathematics Education, 3, 49-56.

[7] Karagiorgi, Y., \& Symeou, L. (2005). Translating Constructivism into Instructional Design: Potential and Limitations. Educational Technology \& Society, 8 (1), 1727.

[8] Lear, J. C. (1993). Barrelled spaces (Order No. EP21603). Available from ProQuest Dissertations \& Theses Full Text; ProQuest Dissertations \& Theses Global. (304084654). Retrieved June 2, 2015 from http://search.proquest.com/docview/304084654

[9] Lear, Jeffrey. (2017). A Systematic Review of Research: A Second Generation Instructional Design Lens on Physics, Autonomy Support, Gender Gap Reduction, and Measuring Mathematics Reasoning Ability. International Journal of Technology and Inclusive Education, 6(1), 1011-1022. DOI: 10.20533/ijtie.2047.0533.2017.0129

[10] Linchevski, L., \& Livneh, D. (1999). Structure sense: The relationship between algebraic and numerical contexts. Educational Studies in Mathematics, 40(2), 173196.

[11] Merrill, M. D., Li, Z., \& Jones, M. K. (1990). Second generation instructional design (ID2). Educational Technology, 30(2), 7-14.

[12] Novotná, J., \& Hoch, M. (2008). How structure sense for algebraic expressions or equations is related to structure sense for abstract algebra. Mathematics Education Research Journal, 20(2), 93-104.

[13] Schoenfeld, A. H. (1988). When good teaching leads to bad results: The disasters of' 'well-taught' mathematics courses. Educational Psychologist, 23(2), 145-166.

[14] Lear, J. (2019). Mathematics Learning Instrument: Development of an Algebra Concept Inventory to Measure Metric Sense Conceptual Understanding. In Proceedings of the Canada International Conference on Education: CICE-2019. Mississauga, Ontario, Canada: Infonomics Society. Retrieved from http://jeffreylear. com/professional/conference/cice/Mathematics_Learning _Instrument_Metric_Sense_LearCICE19_Final.pdf 